\title{
Assessment of radio communication system reliability by system dynamics method
}

\author{
Batzorig BAZARGUR ${ }^{1}$, Otgonbayar BATAA ${ }^{2}$, Uuganbayar BUDJAV ${ }^{3}$ \\ 1,2 Department of Communications Engineering Technology, School of Information and Telecommunication Technology, \\ Mongolian University of Science and Technology, Ulaanbaatar 46/520, Mongolia. \\ ${ }^{3}$ System Thinking Laboratory, Mongolian University of Science and Technology, Ulaanbaatar 46/520, Mongolia. \\ batzorigbazargur@gmail.com, otgonbayar_b@must.edu.mn, buuganaa@must.edu.mn
}

\begin{abstract}
.
Radio communication system in an underground mine is one of the very essential systems for the underground mine. It is required that the radio communication system must be reliable from the starting to construct the underground mine to the closure of the mine. However, underground mine radio communication systems reliability is required to be tested on an active radio communication system in a real environment. In the study was suggested a new research methodology that is studied reliability using dynamic system modeling on Vensim software instead of traditional method to study the reliability of radio communication systems calculating large-scale differential equations. In other words, we suggest a new research methodology. The Motorola Dimetra (TETRA) radio communication system's availability readiness information was used to simulate the reliability of the underground mine radio communication system probability of reliability using Vensim software for system dynamic modeling.

Also, the factors that affect the reliability of underground mining radio communication systems was studied. The study was determined factors that affect the underground mine radio communication system from the following risks. The study was in the examples of the Oyu Tolgoi underground mine. The factors that affect the reliable operation of the underground mine radio communication system were determined using the failure statistics of TETRA radio communication system in the Oyu Tolgoi mine in 2015-2018.
\end{abstract}

\section{Keywords}

Availability, underground mining, radio communication system, influence parameters

\section{Introduction}

Oyu Tolgoi underground mine was as example in the scope of the study and we have determined factors that affect the underground mine radio communication system from the following risks. Among them, the factors influencing the reliable operation of the underground mine radio communication system were determined using failure statistics of the Oyu Tolgoi mine's TETRA radio communication system in 2015-2018. The following are the factors that may affect the reliability of the Oyu Tolgoi underground mine radio communication system.

- Failure of the optical cable (Depending on the condition of the connection, if the main connection is lost, communication with people in the underground will be lost. If the connection was lost in some area of the underground mine, there will be no radio communication in that area)

- Damage of repeater device (Repeater devices are connected and to the base station by optical cables, they will not work if the optical cable is broken. There is also a risk of damage due to any physical damage.)

- Breakage radiation cable (Radiation cable damage (shock, crushing, or cracking)

- Changes and maintenance in the system (various upgrades (hardware and software) on the core system can lead to service interruptions probability)

- Switch to local site trunking mode of TETRA system (in case of disconnection from core system, the base station will work only on its rest network and the user will not be able to call to registered users within the scope of other base stations) 
- Power outage (In the event of a power outage, all core systems, base stations, and underground repeaters will be disrupted)

- Poor connection of radiation cable (delay due to poor connection of radiation cable connectors)

- Interference (close to high voltage cables, close to ventilation fans, etc.)

- Excessive length of radiation cable (more than 800 meters. It is impossible to talk at 1200 meters)

- Return loss problems (power balance problems such as increase or decrease capacity of the repeater, as well as the effect of two different repeaters on each other)

Regarding the research object, based on the information of the Oyu Tolgoi underground mine radio communication system failure in 2015-2018, the causes of the failure were determined by a team of engineers and technicians. In other words, the influence parameters of the reliable operation of radio communication described above have been determined by failure statistics based on information in 2015-2018. Since this information on failure is calculated in minutes, the values of the failure function meaning $(\lambda)$ and the repair function meaning $(\mu)$ were taken as the same in the study. This is because even if the values of the failure function meaning $(\lambda)$ were calculated in minutes, it would be very difficult to take the values of the repair function $(\mu)$ as integers or fractions, which would be the same percentage as the meaning of the failure function $(\lambda)$. Comparing the causes of failure with the system failure time data from the ten reasons listed in Table 1 below, $64.6 \%$ were power line interruptions, $33.6 \%$ were optical line interruptions, $0.0027 \%$ were radiation cable failures, and poor-quality connection of radio cable connector $0.013 \%$ and interference issues $0.00085 \%$ respectively. However, the proportion of the remaining five problems is very small and is not included in the calculation. Earlier, we studied earthquakes at underground mines. In the study, we have classified factors that could affect the reliability of underground radio communication systems, have analyzed seismic distribution using seismic statistics, and have calculated the probability of earthquakes in the underground mine [1]. Another study is on RF repeater isolation and the distribution of radiation cables in tunnels by the complex structure of the underground mine [2] and the study was tested in a real environment. In the study related to human dependent factors of our study, we have developed emergency analysis using Graph Theory [3] and Dijkstra's algorithm developed in the Matlab program and developed the algorithm [4] in the case of an underground mine accident, switching the radio communication service from failure to normal condition, as well as removing the miners quickly from the underground mine. The results of the breakdown using the 4 different distribution functions and the calculation of the average breakdown time show that the resource system is required necessarily to ensure the reliability of the communication system. These results were shown that in the case of underground mining, it is necessary to have a resource radio communication system [5]. The following research section examines in more detail that a radio communication system can be more reliable if it has a resource system. For example, in our article [6], we have improved the reliability section, have developed the Exponential, Poisson, and Weibull distributions for one redundancy (two parallel) and two redundancy ( 3 parallel) radio communication systems, and have presented the results of the readiness function in Matlab. However, during our study on the design of single-resource and double-resource radio communication systems, we have faced the following problems:

Simulating and testing in Matlab requires a lot of programming code, and it was relatively easy to simulate in Vensim.

- When the values of the failure function $(\lambda)$ and the repair function $(\mu)$ of a two-resource radio communication system were modeled by Weibull's distribution law, the reliability was not converged to a fixed number, i.e. it was not stabilized. As a result, three parallel radio communication systems were left in terms of Weibull distribution.

- $\quad$ Also, for one redundancy (two parallel) and two redundancy (3 parallel) radio communication systems we have entered the failure function $(\lambda)$, the repair function $(\mu)$ in mixed form or the failure function $(\lambda)$ on the index, Poisson on the repair function $(\mu)$ or Weibull on the failure function $(\mu)$, etc. This idea was not implemented because the reliability index was not stabilized.

- When we tested modeling of two or three parallel radio communication systems, we used the Dsolve command of the Matlab program. This process was too slow for results and then we have chosen the ode23 command instead of the Dsolve command and we have completed the calculation.

- When the values of the failure function $(\lambda)$ and the correction function $(\mu)$ were assumed to be governed by Poisson's distribution law, the factorial ( $\mathrm{t}$ !) of $\mathrm{t}$ was included in our formula, which made it difficult to calculate differential equations. Therefore, the factorial $(\mathrm{t} !)$ of $\mathrm{t}$ was replaced by the Stirling formula.

- $\quad$ The differential equation systems were omitted in some cases because of recording and calculating complexity in the two-resource radio communication system model when all variants were taken [7]. A basic of the using system dynamic modeling in this study was determined by the above reasons. 


\begin{tabular}{|c|c|c|c|c|c|c|c|c|c|c|c|}
\hline Year & $\begin{array}{l}\text { Interrupted } \\
\text { time }\end{array}$ & $\begin{array}{c}\text { Optical } \\
\text { line } \\
\text { breakage }\end{array}$ & $\begin{array}{c}\text { Changes } \\
\text { and } \\
\text { maintenance } \\
\text { which will be } \\
\text { done on the } \\
\text { system }\end{array}$ & $\begin{array}{l}\text { Switch to } \\
\text { TETRA } \\
\text { system } \\
\text { Local site } \\
\text { trunking } \\
\text { mode }\end{array}$ & $\begin{array}{c}\text { Power } \\
\text { line } \\
\text { interruption }\end{array}$ & $\begin{array}{c}\text { Radiation } \\
\text { cable } \\
\text { failure }\end{array}$ & $\begin{array}{l}\text { Radiation } \\
\text { cable } \\
\text { connection } \\
\text { problems }\end{array}$ & Interference & $\begin{array}{l}\text { Exceeding } \\
\text { the length } \\
\text { of the } \\
\text { radiation } \\
\text { cable }\end{array}$ & $\begin{array}{c}\text { Return } \\
\text { loss } \\
\text { problem }\end{array}$ & $\begin{array}{l}\text { Repeater } \\
\text { equipment } \\
\text { failure }\end{array}$ \\
\hline 2015 & 15820 & 7483 & 8 & 8 & 7215 & 632 & 411 & 20 & 34 & 6 & 3 \\
\hline 2016 & 211796 & 2317 & 12 & 12 & 178219 & 2631 & 28650 & 46 & 72 & 15 & 2 \\
\hline 2017 & 978779 & 397135 & 27 & 27 & 579625 & 1471 & 348 & 61 & 56 & 24 & 5 \\
\hline 2018 & 959076 & 321561 & 31 & 31 & 635643 & 1254 & 421 & 59 & 51 & 17 & 8 \\
\hline Total & 2165471 & 728496 & 78 & 78 & 1400702 & 5988 & 29830 & 186 & 213 & 62 & 18 \\
\hline & $\mu$ & 0.3363866 & $3.60 \mathrm{E}-05$ & $3.60 \mathrm{E}-05$ & 0.646781 & 0.002765 & 0.01377415 & $8.59 \mathrm{E}-05$ & $9.84 \mathrm{E}-05$ & $2.86 \mathrm{E}-05$ & $8.31 \mathrm{E}-06$ \\
\hline & $\lambda$ & 0.3363866 & $3.60 \mathrm{E}-05$ & $3.60 \mathrm{E}-05$ & 0.646781 & 0.002765 & 0.01377415 & $8.59 \mathrm{E}-05$ & $9.84 \mathrm{E}-05$ & $2.86 \mathrm{E}-05$ & 8.31E-06 \\
\hline
\end{tabular}

Tab. 1. Oyu Tolgoi underground mine failure statistics information in 2015-2018.

\section{Reliability analysis of underground mining radio communication systems using dynamic modeling}

The papers on the dynamic modeling system were related to reasons for the repairs, the operating and maintenance costs, the planning of the repair work, the repair work costs, and improving the completion [8-11]. We have introduced to the papers on disaster management [12], and creating paradoxical effects of an abnormal social phenomenon [13], mixed models [14], experimental methods [15], analysis [16], and the development stages of the system dynamics modelling methodology [17], also evaluation effectiveness of educational training [18], the correlation between the various components [19], and have developed a correlation between safety factors [20], to calculate the reliability [21], classified researches and studies on system dynamic modelling [22]. We have not found any paper on using the system dynamic modelling methodology for studying reliability of underground radio communication systems. In this study we have introduced results of the probability of trouble-free operation of the Motorola Dimetra radio communication system in the underground mine which was tested and simulated by system dynamic modeling. In this study, the dynamic modeling of the system was modeled for three core systems: MSO (Mobile Switching Office) and one BTS, one OMU device, and a two-repeater device. As for our system dynamic modeling there were not included all 25 base stations and related to them transmitters, that 200 times but were modeled only three core systems as MSO and one BTS, one branch OMU that tap from it, for duplicated transmitters. The form of the differential equation is according to Weibull's distribution law:

$$
\frac{d P}{d t}=\mu \beta t^{\beta-1}
$$

The main reason for choosing this methodology was the need to consider calculating at least twenty-six differential equations for modeling twenty-six devices which were described in Figure 1. Because of the need to calculate at least twenty-six differential equations we have chosen easier and more obvious system dynamic modeling. The probability of reliable operation of the underground mining radio communication system was studied in the following steps:

1. Structuring the radio communication system.

2. Entering information on the reliability of the radio communication system manufacturer.

3. Collecting statistical information on the failure of the radio communication system.

4. Identifying factors that affect the reliability of the radio communication system.

5. Selecting the form of the differential equation based on the failure statistics (in our case $\frac{d P}{d t}=\lambda_{1} \beta t^{\beta-1} e^{(-t)^{\beta}}$

the Weibull distribution function is expressed by the differential equation). 


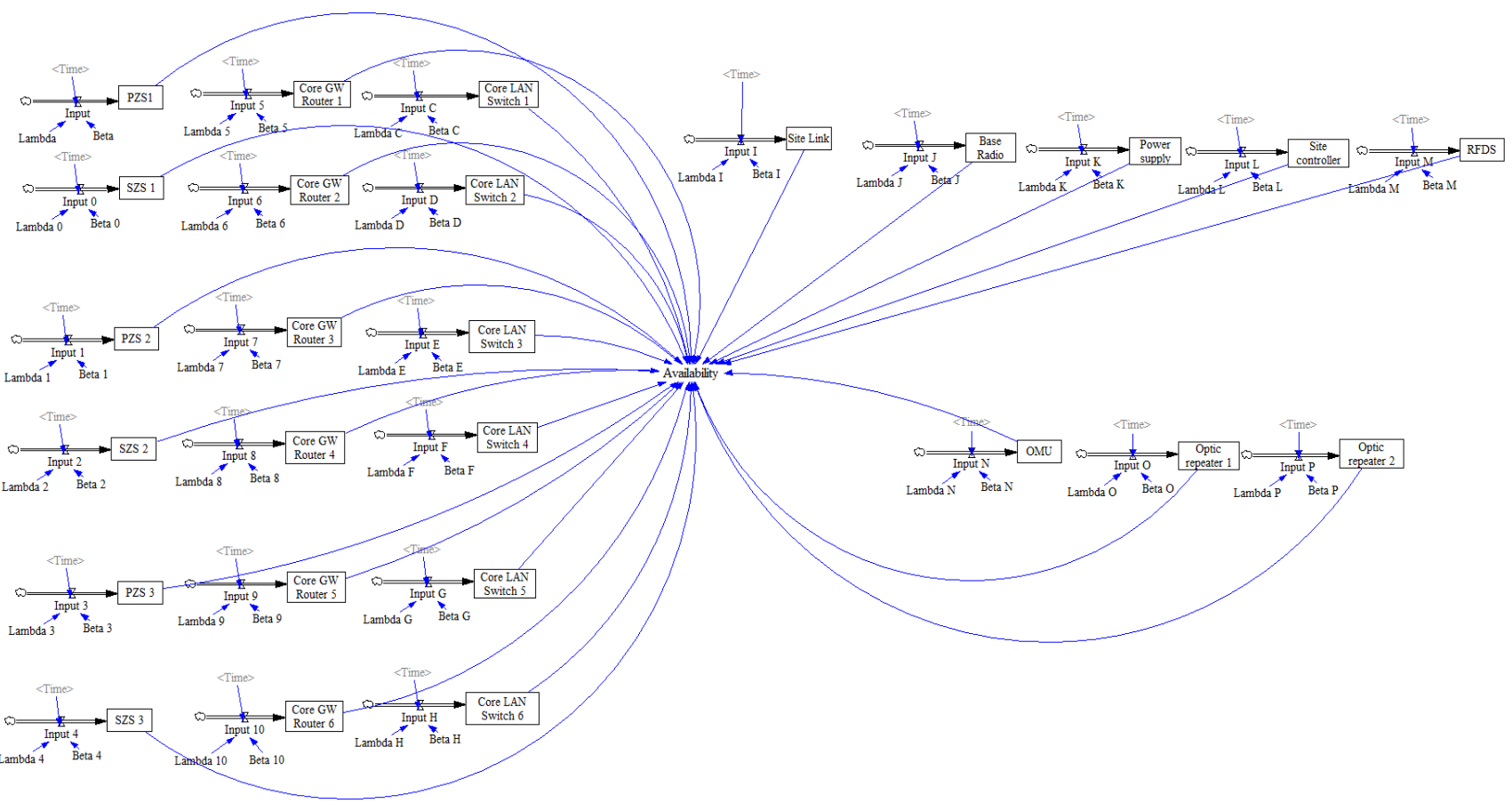

Fig. 1. Reliability model of underground mining radio communication system using Vensim software

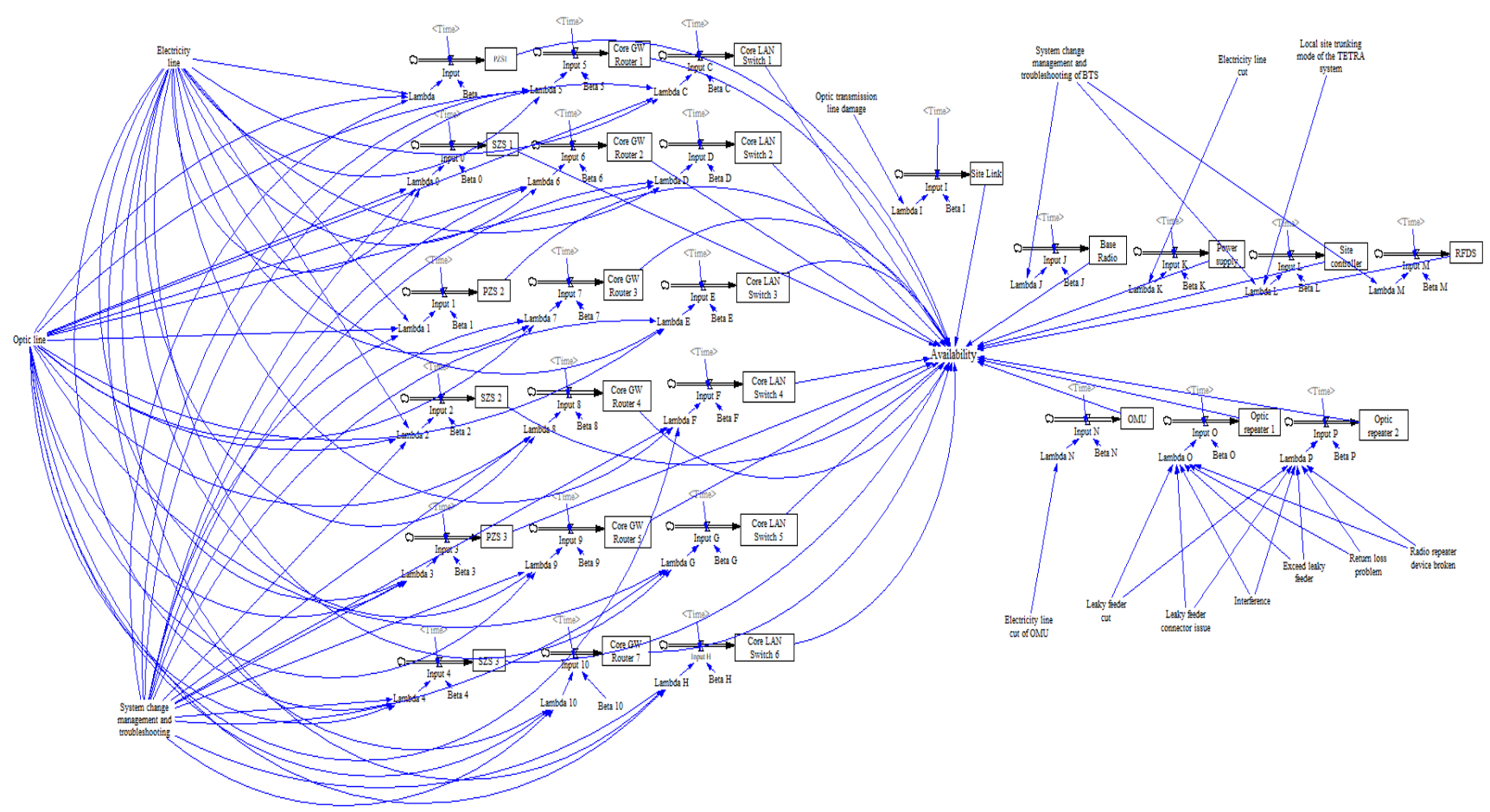

Fig. 2. Reliability model of underground mining radio communication system, where was considered the influence parameters 


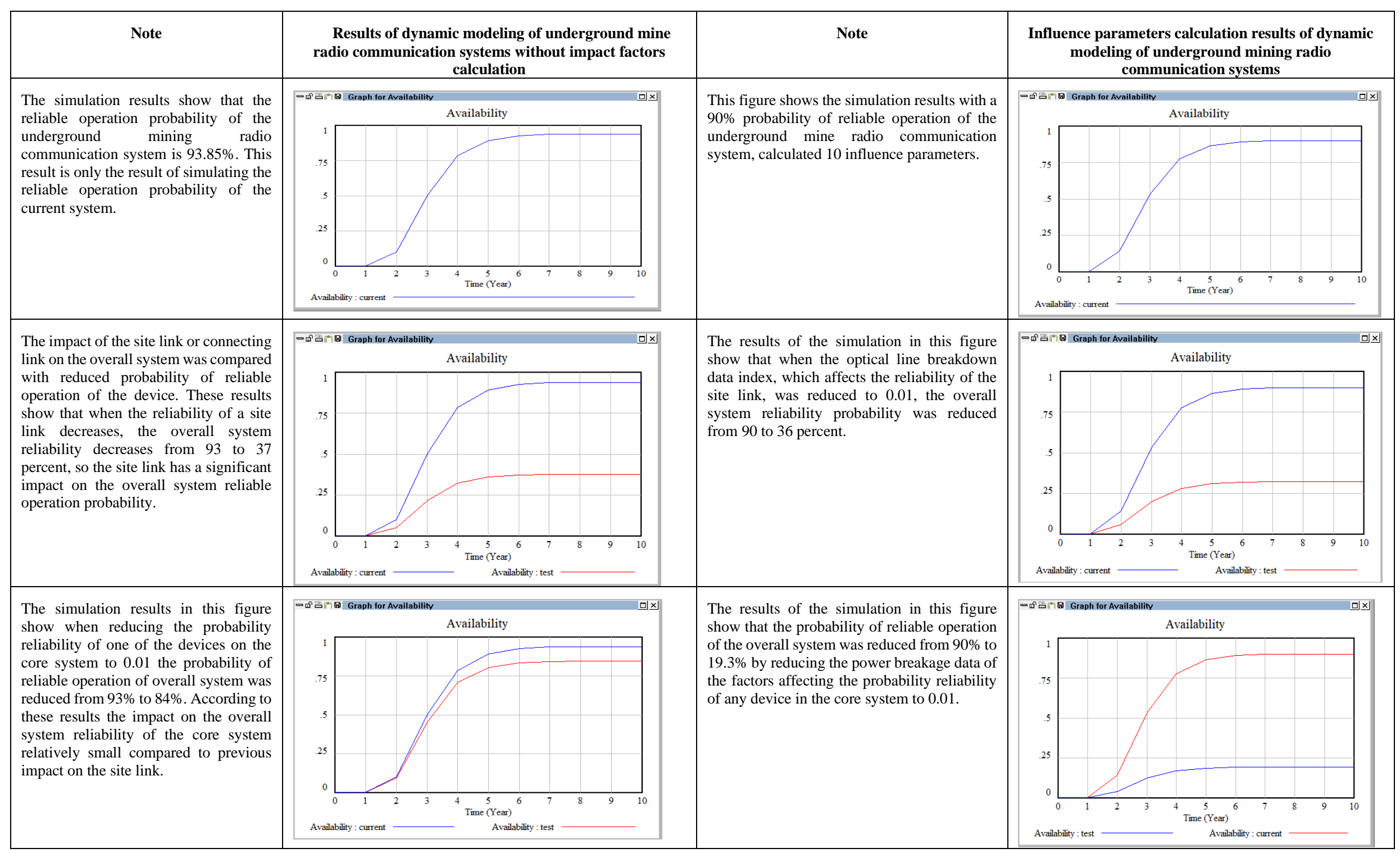

Tab. 2. Results of the study 
6. After that, was selected the program that calculates the differential equation, and in our case, was chosen the Vensim program.

7. Developing a radio communication system model on Vensim software.

8. Making a simulation and testing it.

9. Showing end results.

In the model of the underground mine radio communication system, where was considered the impact factors, and was developed in Vensim software using the following weighting function method:

$P=\frac{P_{1} P_{2} P_{3}+P_{4} P_{5} P_{6}}{2}+\frac{P_{7} P_{8} P_{9}+P_{10} P_{11} P_{12}}{2}+\frac{P_{13} P_{14} P_{15}+P_{16} P_{17} P_{18}}{2} * P_{19} P_{20} P_{21} P_{22} P_{23} P_{24} * \frac{P_{25}+P_{26}}{2}$

\section{Conclusion}

We have studied influence parameters and the reliability of underground radio communication systems in the underground mine and have calculated their maximum impact. Based on these probabilities of reliable operation of the underground radio communication system was studied by simulation using system dynamics modeling. The results of the studies on system dynamic modeling for the probability of reliable operation of the radio communication system and the impact factors for the reliability of the system were introduced. Also, the devices included in the radio communication system were modeled using software Vensim by simulating information or parameters of the probability of the manufacturer's device's reliable operation. First, we have simulated entering probability reliability indexes or $\lambda$ meaning in the model where have not been taken affecting factors and the reliable operation availability parameters provided by the manufacturer of each Motorola Dimetra system devices and each Cobham repeater devices. By that, the initial meaning of each device was entered variously and the probability of reliable operation of the overall system, i.e. optimization, was modeled to the highest possible meaning. As Table 2 shows, the site link or connecting link between the Core system and the base station was the part that had the greatest impact on the probability of reliable operation of the underground mine radio communication system.

However, after testing the 10 influence parameters, the affecting factors were added to the core system, site link, BTS (base station), and for $\lambda$ meaning relating to each repeater device. The affecting factors were tested one by one and they have been designed. As a result of the simulation after this modeling, it is clear from Table 2 that the power failure factor affecting the core system device has a greater effect on the reliability of the system than the site link. In other words, during simulation for affecting factors for reliable operation we have proved that it is necessary operation failure for overall system when power failure in core system. The most important factors affecting the reliability of the system were power breakage $(64.6 \%)$ and optical transmission line $(33.6 \%)$, and effects of these factors were simulated. The results of the simulation are shown in Table 2.

- The structure of the radio communication system reliability study considered in this structure can be applied to any component radio system.

- Also, it is possible to enter system readiness parameters for any radio communication system provided by the manufacturer and to develop it using this model.

- It is possible to enter and to design the impact parameters of the influence parameters of a radio communication system.

- In the future, our study is possible to study probability of reliable operation of radio systems of other types of radio systems (not only TETRA, but also analogue conventional, LTE, 5G, etc.) and influence parameters, also it is possible to expand the study. It can also be used not only for radio systems, but also for the reliability of underground heating networks and sewer networks and to calculate the influence parameters of those networks. The novelty of the study is that it differs from traditional research methods by considering the probability of reliable operation of the underground mining radio communication system using the system dynamics method and the influence parameters of the reliable operation of this system. Also, the study was more optimized. In other words, the main methodology of the study was the system dynamic method. The main assumption we expected from the study was that the probability of reliable operation of the system would be at least $90 \%$. As the assumption was $93.85 \%$ and our initial hypothesis was confirmed. 


\begin{abstract}
About the Authors ...
Batzorig BAZARGUR was born in Choibalsan city, Dornod aimag, Mongolia. He graduated from the Mongolian Railway Institute in 2009 with bachelor's degree. In 2010, completed master's degree program of the Mongolian University of Science and Technology. Research topic for PhD degree is "Factors affecting reliability of underground mine radio communication system".

Otgonbayar BATAA (corresponding author) was born in Ulaanbaatar city, Mongolia. Bataa Otgonbayar, in 1978 graduated from the Polytechnic Institute of Mongolia majoring in Radio communication engineer. Bachelor's degree thesis: "Feasibility study of improving the efficiency of discrete information system". Master degree thesis (M.Sc) in 1995:"Some issues of speech synthesis". PhD degree thesis in 1996:" Study of Mongolian speech synthesis and applying it in telecommunication technics", in 2003, post Ph.D program thesis: "Optimal version of OFDM system frequency and time-distortion". Professor. Consulting engineer of Mongolia. Research topic:" Broadband, high speed integrated services technologies” (WiMAX, WiBro, Mobile IPTV, LTE etc).

Uuganbayar BUDJAV (corresponding author) was born in Undurshil soum, Dundgovi aimag, Mongolia. Bachelor's degree in the National University of Mongolia, in 1998. Master's degree in the National University of Mongolia, in 2000. In 2009, PhD degree in the Mongolian University of Science and Technology. Chief at the System Thinking Laboratory, Mongolian University of Science and Technology. Research topic:" Non-linear dynamics model, system dynamics model".
\end{abstract}

